\title{
RECHERCHE ET DOSAGE DU DIACÉTYLE DANS LE BEURRE PRODUIT DANS LA PROVINCE DE PÉROUSE (1)
}

\author{
par \\ ManLIo BROGIONI
}

La présence de diacétyle dans le beurre a fait et fait encore actuellement l'objet d'affirmations contradictoires, de sorte que le problème de son existence naturelle ou de son adjonction pour conférer au beurre son arome ou pour l'améliorer, conserve à beaucoup d'égards l'incertitude du début.

On sait que, tout en subissant l'influence de nombreux facteurs, le diacétyle est produit par certaines fermentations, comme les fermentations lactique, acétique, etc., et que, par conséquent, il doit logiquement se trouver dans du beurre, produit dans des conditions normales, compte tenu du milieu naturel dont ce beurre provient.

Déjà eñ 1931-1932, Testoni et Ciusa [1], travaillant sur quelques beurres italiens, ne trouvèrent que rarement du diacétyle, d'où ils conclurent que sa présence observée sporadiquement devait être attribuée à des causes accidentelles.

Les auteurs utilisèrent pour leurs recherches la méthode au carbonate d'hydroxylamine et sulfate de nickel, avec solubilisation de la nickeldiméthylglyoxime d'après FeIGL [12].

Puis des chercheurs français et allemands, parmi lesquels Schmalfuss [2], Barnicoat [4], Brioux [6], Cox et Wriex [7], De Bucca [9], Dehove [8], Mahler et Helberg [3], Pien [5], et d'autres, démontrèrent au contraire la présence de diacétyle dans les beurres fabriqués dans leurs pays respectifs, et considérèrent en conséquence qu'il représentait un constituant normal de formation biologique et qu'il était en outre l'indice d'une bonne fabrication.

En 1937, PIEN [5] abandonna la méthode au diméthylglyoximate dè nickel jusque-là utilisée par tous les autres chercheurs ; il effectua de nombreuses recherches sur le réactif à la $p$. toluylènediamine avec laquelle il obtint une quinoxaline de couleur jaune et indiqua que ce réactif était plus sensible et plus pratique que le premier. Il devait cependant reconnaître l'impossibilité d'effectuer un dosage précis du diacétyle s'il n'était pas opéré sur un kilogramme de beurre ou tout au moins sur 250 grammes.

En raison de ces graves inconvénients, et considérant que dans

(1) Communication présentée par M. Pien, à la séance de la Société des ExpertsChimistes de France, le 10 novembre 1954. Ann. Fals. Fr., no 554, 56, 1955. 
la combinaison diacétyle-diamine, l'introduction de plus nombreux noyaux benzéniques augmente l'intensité de la coloration. PIEN prépara lui-même le chlorhydrate de tétraminodiphényle, qui, en présence de quantités même minimes de diacétyle, donne des réactions très sensibles, par formation d'une diphénylquinoxaline intensément colorée en jaune. On pourrait, à l'aide de cette réaction, mener les recherches avec sécurité, en employant des quantités de beurre de 50 à 100 grammes au maximum.

PIEN indiqua en outre que la plupart des beurres français contenaient jusqu'à 0 mgr. 5 de diacétyle par kilogramme, tandis que peu en contenaient jusqu'à 1 milligramme et quelques exceptions de 1 à 2 milligrammes par kilogramme.

En 1937, BRIoux et Jours [6] abandonnant également la méthode à la diméthylglyoxime et travaillant avec le réactif au tétraminodiphényle proposé par Pien, conclurent, après une série de recherches sur un nombre considérable de beurres frais de Normandie, que ces derniers contenaient normalement du diacétyle $\grave{a}$ une teneur comprise entre 0 mgr. 05 et 0 mgr. 5 par kilogramme, et allant dans certains cas jusqu'à $1 \mathrm{mgr}$. 5 et exceptionnellement même jusqu'à 2 mgr. 5 .

De même, en 1948, Holw' ERDA et VAN DER GeEst [10] démontrèrent que les produits de la meilleure qualité obtenus à partir de crèmes douces, pouvaient également atteindre une teneur de $1,2 \mathrm{mgr} . / \mathrm{kg}$. de diacétyle.

Inversement, CUPPINI, toujours en 1948, étudiant les beurres italiens, à l'aide de la réaction à l'hydroxylamine et au sel de nickel, n'observa pas la présence de diacétyle qu'il trouva par contre dans le beurre de sérum, ou ayant reçu une adjonction de sérum.

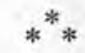

J'ai done pensé qu'il ne serait pas inutile de procéder à la recherche, et éventuellement au dosage, du diacétyle sur le beurre actuellement produit dans la province de Pérouse, sur lequel, à ma connaissance, aucune recherche de ce genre n'a été effectuée.

Les échantillons de beurre, toujours de fabrication récente, employés pour l'analyse, provenaient du traitement normal direct du lait effectué dans les beurreries existant dans la province, soit des localités de Ponte San Giovanni dans la commune de Pérouse, Santa Lucia dans la commune de Pérouse, Santa Maria degli Angelì dans la commune d'Assise, San Valentino dans la commune de Marsciano.

En vue de déceler et éventuellement de doser des quantités 
minimes de diacétyle, il m'a paru avant tout nécessaire de vérifier dans leur application pratique les méthodes jusqu'ici employées et proposées, afin de conférer aux résultats obtenus la plus grande certitude possible.

J'ai donc procédé aux recherches en utilisant des solutions étalonnées, préparées extemporanément de diacétyle, absolument pur, à des concentrations de 0,05 à 1 milligramme par $100 \mathrm{~cm}^{3}$, en travaillant toujours sur $20 \mathrm{~cm}^{3}$ de solution, e'est-à-dire sur une quantité identique à celle $\mathrm{du}$ distillat que j'ai obtenu en utilisant 50 grammes de beurre, comme je le décrirai par la suite.

Les méthodes examinées furent :

10 Celle à la p. toluylènediamine ;

$2^{\circ}$ Celle à l'hydroxylamine et sulfate de nickel ;

$3^{\circ}$ Celle au chlorhydrate de tétraminodiphényle.

La première méthode de Pren à la p. toluylènediamine, bien que spécifique, possède une faible sensibilité. Des concentrations de $0 \mathrm{mgr} .1$ par $100 \mathrm{~cm}^{3}$ ne font pas apparaître de coloration qui puisse être appréciée.

La méthode de l'hydroxylamine et sulfate de nickel, également spécifique, a été éprouvée par mes soins dans les nombreuses variantes proposées par divers auteurs; ces variantes, tout en augmentant la sensibilité de la méthode originale, ne permettent pas toutefois d'obtenir des résultats satisfaisants à des concentrations de $0 \mathrm{mgr}$. 15 par $100 \mathrm{~cm}^{3}$ de diacétyle, pour lesquelles la formation de quelques cristaux de diméthylglyoximate de nickel n'est plus appréciable que par la coloration rouge carmin de ce composé. A de telles concentrations, on peut encore en suivant la technique décrite par Dehove et Dessirier [8], déceler le composé, mais son dosage colorimétrique proprement dit est aléatoire et incertain.

La méthode, longue et pénible, par suite de nombreuses et délicates opérations à effectuer, n'est pas adaptée pour l'analyse des beurres, ni d'application facile lorsque, comme je l'ai fait pour essayer d'accélérer son exécution, on procède, après concentration de la solution, à la séparation par centrifugation des cristaux de diméthylglyoximate et à leur solubilisation dans le tube même de la centrifugeuse.

De plus, il est nécessaire pour atteindre les limites de sensibilité, de la méthode d'effectuer l'extraction du diacétyle à partir de quantité de beurre qui ne soient pas inférieures à 300 grammes au moins, ce qui représente un inconvénient évident et peut toujöurs donner lieu à des résultats analytiques négatifs, en raison des très petites quantités de diacétyle qui ne peuvent ainsi être décelées.

La méthode imaginée et proposée par PIEN, au chlorhydrate 
de tétraminodiphényle, en plus de sa spécificité, est d'une extrême sensibilité et permet d'effectuer des dosages colorimétriques exacts et rapides, même dans des solutions contenant des concentrations inférieures à $0 \mathrm{mgr}$. 05 par $100 \mathrm{~cm}^{3}(0,5 \%$ o) .

Cette réaction, toute simple, consiste uniquement à introduire dans le distillat une solution de chlorhydrate de tétraminodiphényle, avec lequel il se forme en présence de diacétyle une diphénylquinoxaline de couleur jaune, que l'on peut parfaitement comparer colorimétriquement à des standards obtenus par dilution d'une solution de bichromate de potassium à $1 \%$.

L'unique inconvénient, si l'on peut dire, de cette méthode, est celui d'être obligé de préparer le chlorhydrate de tétraminodiphényle que l'on ne trouve pas dans le commerce (1). J'ai effectué cette préparation en me conformant rigoureusement aux indications de Pien [5] et en partant de la benzidine. On obtient un produit pur, extrêmement soluble, pouvant être conservé longtemps dans un flacon bien fermé et à l'abri de la lumière.

D'après Pien, la solution du réactif doit être préparée extemporanément, car elle s'oxyde facilement, brunit, et devient inutilisable (2).

J'ai cependant pu constater qu'en traitant la solution oxydée par du charbon de bois activé, et en la filtrant, on obtient à nouveau une solution incolore, qui donne de la même façon la coloration jaune caractéristique en présence de diacétyle.

A la suite de ce que je viens d'exposer, j'ai done adopté pour la recherche et le dosage du diacétyle dans les beurres mentionnés plus haut, la méthode au chlorhydrate de tétraminodiphényle, qui me paraissait convenir sous tous les rapports.

$$
\text { *** }
$$

Pour l'extraction du diacétyle, j'ai abandonné la méthode à l'alcool éthylique, déjà décrite par GuILlot et VIzerN, parce qu'elle n'aboutit jamais à l'extraction de la totalité du composé, et j'ai utilisé la méthode d'extraction par la vapeur d'eau, qui entraîne la totalité du diacétyle présent dans le beurre, ce qui confirme les résultats de Dehove et Dessirier [8].

Je me suis servi d'un appareil ainsi construit pour réaliser cette opération :

(1) On peut maintenant se procurer ce réactif, en s'adressant à la Maison Prolabo, 12, rue Pelée, à Paris (J, P.).

(2) La préparation du réactif ayant été amélioré,, celui-ci serait maintenant parfaitement stable et resterait blanc, même après plusieurs années de conservation (5 b.). 
- Un générateur de vapeur, formé d'un ballon de verre à fond plat et col long, muni d'un bouchon à deux trous, l'un pour le tube d'amenée de la vapeur, l'autre pour un tube de verre avec robinet à l'émeri, permettant de régler la marche de la distillation, soit au début, soit ensuite, et d'éviter qu'à la fin, au moment de son ouverture, le beurre employé pour l'extraction ne soit aspiré à travers le tube d'adduction.

- Un ballon de verre Iéna, d'une contenance de $250 \mathrm{~cm}^{3}$, à fond rond, col large et court, muni d'un bouchon à deux trous, par l'un desquelles passe le tube d'amenée de vapeur, et par l'autre le tube de verre à travers lequel la vapeur transportant le diacétyle est amenée à un réfrigérant de 30 centimètres environ, dont l'extrémité inférieure, convenablement effilée, baigne dans un petit bécher de $50 \mathrm{~cm}^{3}$ environ, dans lequel on introduit au départ $5 \mathrm{~cm}^{3}$ d'eau distillée, pour éviter les pertes de diacétyle.

J'ai donné à l'extrémité du tube d'injection de vapeur, qui doit atteindre le fond du ballon de distillation, la forme d'une sphère plutôt grande, munie de fines et nombreuses ouvertures, afin de répartir l'action et la pénétration de la vapeur dans tous les sens, en produisant en outre, avec la faible énergie de propulsion de la vapeur elle-même, un brassage rapide et continu du beurre fondu.

- Un bain d'huile de vaseline.

On peut décrire ainsi le mode d'extraction :

On introduit 50 grammes de beurre dans le ballon qui, immergé dans le bain d'huile, reste maintenu à une température comprise entre $105^{\circ}$ et $110^{\circ}$, pendant toute la durée de l'extraction. Cette température ne doit en aucun cas être dépassée, pour les raisons qui seront indiquées plus loin. Avant d'admettre la vapeur et de commencer la distillation, le ballon et son contenu doivent atteindre la température du bain, ce qui demande à peu près 4 à 5 minutes.

On distille ainsi dans le petit bécher jusqu'à ce qu'on obtienne, avec les $5 \mathrm{~cm}^{3}$ d'eau distillée mise au départ, un volume de $20 \mathrm{~cm}^{3}$. Il est nécessaire enfin de conduire la distillation et de régler l'émission de vapeur de telle sorte que l'on obtienne un distillat parfaitement froid.

En opérant ainsi, j'ai constaté que la distillation de $15 \mathrm{~cm}^{3}$ de liquide est plus que suffisante pour entraîner tout le diacétyle présent; des recherches faites sur des fractions ultérieures du distillat furent complètement négatives.

Sur le distillat obtenu, on pratique la réaction de la manière suivante:

Dans le même verre, contenant les $20 \mathrm{~cm}^{3}$ de liquide, on ajoute $0 \mathrm{~cm}^{3} 75$ de la solution de chlorhydrate de tétraminodiphényle à 
$2,5 \%$, en agitant, puis $0 \mathrm{~cm}^{3} 75$ d'acide chlorhydrique concentré, d'après les indications données par l'auteur de la méthode.

On obtient une nette coloration jaune plus ou moins intense, suivant la quantité de diacétyle présent, par formation de diphénylquinoxaline. On procède au dosage colorimétrique du diacétyle à l'aide du photomètre gradué de Zeiss, filtre L 3.

La méthode, non seulement donne une solution colorée parfaitement stable pendant 24 heures et plus, mais elle ne présente de difficultés d'aucune sorte, et réduit au minimum les opérations analytiques, de sorte que l'on peut distiller le diacétyle et le doser en 30 minutes environ.

On peut utiliser comme standard soit une solution de diacétyle de titre connu, soit plus simplement une solution obtenue par dilution d'une solution mère de bichromate de potassium à $1 \%$, toujours d'après les indications de l'auteur de la méthode.

Il est de la plus grande importance, comme je l'ai indiqué, de maintenir la température du bain d'huile pendant la distillation à $110^{\circ}$, car à des températures plus élevées ou bien le distillat ne présente plus la réaction (dans le cas de petites quantités de diacétyle), ou bien cette réaction est bien moins intense. Cela amènerait à la conclusion que le diacétyle doit subir, à des températures supérieures à $110^{\circ}$, des modifications à la suite desquelles il ne se forme plus de diphénylquinoxaline. J'essaierai, si possible, d'éclaircir cette question au cours de mes prochaines recherches.

Les résultats obtenus sur les échantillons de beurre examinés à l'aide de la méthode indiquée ci-dessus, sont les suivants :

\section{Echantillons No}

Beurrerie de Ponte S. Giovanni .... Beurrerie S. Lucia sobborghi ...... Beurrerie S. Maria degli Angeli .... Beurrerie S. Valentino ............
Diacétyle en mgr. par kg. de beurre

$\begin{array}{cll}1 & 2 & 3 \\ 0.6 & 0.5 & 0.8 \\ 0.7 & 0.7 & 0.55 \\ 0.5 & 0.46 & 0.6 \\ 0.6 & 0.7 & 0.65\end{array}$

\section{CONCLUSIONS}

Tenant compte des résultats analytiques ci-dessus, il est permis d'affirmer que les beurres fabriqués dans la province de Pérouse et obtenus par écrémage du lait par centrifugation, contiennent du diacétyle comme constituant naturel.

La méthode de Pien à la diphénylquinoxaline s'est révélée être la seule valable et applicable à la recherche et au dosage du diacétyle présent dans les beurres en petite quantité. Cette méthode simple, rapide, précise, permet surtout d'opérer sur de faibles quantités de beurre, dont on dispose normalement dans les laboratoires de contrôle. 
Les recherches et dosages du diacétyle, d'après la méthode décrite font actuellement l'objet d'études que je poursuis sur les beurres italiens du commerce et sur des échantillons de provenance connue. Je rendrai compte des résultats obtenus dans une prochaine note.

(Laboratorio Provinciale d'Igiene e Profilassi, Reparto Chimico, Perugia.)

\section{BIBLIOGRAPHIE}

[1] Testoni et Ciusa. Dosamento del diacetile nel burro. Ann. Chim. App. 1931, 21, 3, 147. Origine del diacetile nel burro. Id. id., 1932, $22,1,44$. Ancora del diacetile nel burro. $I d . i d ., 1935,25,1,39$.

[2] Schmalfuss et Niel. Il diacetile nel burro. Physiol. Chemie, 1928, 176.

[3] Mohler et Helberg. Recherche du diacétyle. Mitt. Lebens. Hyg., $1933,24,274-276$.

[4] Barnicoat. Le dosage du diacétyle de l'acétylméthylearbinol. Analyst, $1935,60,715,653-662$.

[5] J. Pien, J. Baisse et R. Martin. Le dosage du diacétyle dans les beurres. Le Lait, 1937, 17, 673,

[5a] J. Pren. La Chimie de l'arômə du bəurre. Ann. Falsifications, 1937, 30,387 et 473 .

[5b] J. Pien. Sur le mierodosage du diaé́tyle dans les bəurras. Id. id., 1948, 41, 529 et $L_{3}$ Lxit, 1949, 288, 461.

[6] Brioux et Jouis.Le diacétyle dans les beurrez normands. Ann. Falsifications, 1937, 39, 457 .

[7] Cox et Wiley. Disage colorimétrique du diacétyle et de l'acétoïne dans les produits laitiers. J. Conneil SC. Ind. Research, 1939, 12, 3, 227-231.

[8] Dehove et Dessirier. Rэchorche et dosage du diacétyle dans les beurres. Ann. Falsifications, 1937, 30, $28 \overline{8}$.

[9] De Buccar. La recherche du diacétyle dans les beurres. Ann. Hyg., $1940,18,7,271-276$.

[10] Holwerda et VAN DER Geest. L'influence de la teneur en diacétyle sur l'odeur et la saveur du beurre. Nederlands Melk-en Zuivelt, 1948, $2,1,25-39$.

[11] Cupprnr. Il diacetile nei burri. Oleria, 1948, 3, 154.

[12] Feigl, Ber., 57, 758, 1924. 